\title{
Identification of tumor microenvironment-associated immunological genes as potent prognostic markers in the cancer genome analysis project HOPE
}

\author{
RYOTA KONDOU $^{1 *}$, YASUTO AKIYAMA ${ }^{1 *}$, AKIRA IIZUKA ${ }^{1}$, HARUO MIYATA ${ }^{1}$, CHIE MAEDA $^{1}$, \\ AKARI KANEMATSU $^{1}$, KYOKO WATANABE ${ }^{1}$, TADASHI ASHIZAWA ${ }^{1}$, TAKESHI NAGASHIMA ${ }^{2,3}$, \\ KENICHI URAKAMI ${ }^{2}$, YUJI SHIMODA ${ }^{2,3}$, KEIICHI OHSHIMA ${ }^{4}$, AKIO SHIOMI ${ }^{5}$, YASUHISA OHDE ${ }^{6}$, \\ MASANORI TERASHIMA $^{7}$, KATSUHIKO UESAKA ${ }^{8}$, TETSURO ONITSUKA ${ }^{9}$, SEIICHIRO NISHIMURA ${ }^{10}$, \\ YASUYUKI HIRASHIMA $^{11}$, NAKAMASA HAYASHI ${ }^{12}$, YOSHIO KIYOHARA ${ }^{13}$, YASUHIRO TSUBOSA ${ }^{14}$, \\ HIROHISA KATAGIRI $^{15}$, MASASHI NIWAKAWA ${ }^{16}$, KAORU TAKAHASHI ${ }^{17}$, HIROYA KASHIWAGI ${ }^{18}$, \\ MASAHIRO NAKAGAWA ${ }^{19}$, YUJI ISHIDA ${ }^{20}$, TAKASHI SUGINO ${ }^{21}$, AKIFUMI NOTSU $^{22}$, \\ KEITA MORI $^{22}$, MITSURU TAKAHASHI ${ }^{15}$, HIROTSUGU KENMOTSU ${ }^{23}$ and KEN YAMAGUCHI ${ }^{24}$ \\ Divisions of ${ }^{1}$ Immunotherapy and ${ }^{2}$ Cancer Diagnostics Research, Shizuoka Cancer Center Research Institute, \\ Shizuoka 411-8777; ${ }^{3}$ Special Reference Laboratory, Tokyo 191-0002; ${ }^{4}$ Medical Genetics Division, \\ Shizuoka Cancer Center Research Institute; Divisions of ${ }^{5}$ Colon and Rectal Surgery, \\ ${ }^{6}$ Thoracic Surgery, ${ }^{7}$ Gastric Surgery, ${ }^{8}$ Hepato-Biliary-Pancreatic Surgery, ${ }^{9}$ Head and Neck Surgery, \\ ${ }^{10}$ Breast Surgery, ${ }^{11}$ Gynecology, ${ }^{12}$ Neurosurgery, ${ }^{13}$ Dermatology, ${ }^{14}$ Esophageal Surgery, ${ }^{15}$ Orthopedic Oncology, \\ ${ }^{16}$ Urology, ${ }^{17}$ Breast Oncology Center, ${ }^{18}$ Ophthalmology, ${ }^{19}$ Plastic and Reconstructive Surgery, ${ }^{20}$ Pediatrics and \\ ${ }^{21}$ Pathology; ${ }^{22}$ Clinical Trial Coordination Office; ${ }^{23}$ Division of Thoracic Oncology, Shizuoka Cancer Center Hospital; \\ ${ }^{24}$ Shizuoka Cancer Center, Shizuoka 411-8777, Japan
}

Received February 16, 2021; Accepted August 5, 2021

DOI: $10.3892 /$ mco.2021.2395

\begin{abstract}
Project High-tech Omics-based Patient Evaluation (HOPE), which used whole-exome sequencing and gene expression profiling, was launched in 2014. A total of $\sim 2,000$ patients were enrolled until March 2016, and the survival time was observed up to July 2019. In our previous study, a tumor microenvironment immune type classification based on the expression levels of the programmed death-ligand 1 (PD-L1) and CD8B
\end{abstract}

Correspondence to: Dr Yasuto Akiyama, Division of Immunotherapy, Shizuoka Cancer Center Research Institute, 1007 Shimonagakubo, Nagaizumi, Sunto, Shizuoka 411-8777, Japan E-mail: y.akiyama@scchr.jp

*Contributed equally

Abbreviations: PD-1, programmed death-1; PD-L1, programmed death-ligand 1; WES, whole-exome sequencing; GEP, gene expression profiling; SNV, single nucleotide variant; CTL, cytotoxic $\mathrm{T}$ lymphocytes; TME, tumor microenvironment; TMB, tumor mutational burden; OST, overall survival time

Key words: TME, immune response-associated gene, prognostic marker, immune type classification, project High-tech Omics-based Patient Evaluation genes was performed based on four types: A, adaptive immune resistance; $\mathrm{B}$, intrinsic induction; $\mathrm{C}$, immunological ignorance; and $\mathrm{D}$, tolerance. Type $\mathrm{A}\left(\mathrm{PD}-\mathrm{L1}^{+}\right.$and $\left.\mathrm{CD} 8 \mathrm{~B}^{+}\right)$exhibited upregulated features of $\mathrm{T}$ helper 1 antitumor responses. In the present study, survival time analysis at 5 years revealed that patients in type A had a better prognosis than those in other categories [5 year survival rate (\%); A (80.5) vs. B (73.9), $\mathrm{C}$ (73.4) and $\mathrm{D}$ (72.6), $\mathrm{P}=0.0005]$. Based on the expression data of 293 immune response-associated genes, 62 specific genes were upregulated in the type A group. Among these genes, 18 specific genes, such as activated effector T-cell markers (CD8/CD40LG/GZMB), effector memory T-cell markers (PD-1/CD27/ICOS), chemokine markers (CXCL9/CXCL10) and activated dendritic cell markers (CD80/CD274/SLAMF1), were significantly associated with a good prognosis using overall survival time analysis. Finally, multivariate Cox proportional hazard regression analyses of overall survival demonstrated that four genes (GZMB, HAVCR2, CXCL9 and CD40LG) were independent prognostic markers, and GZMB, CXCL9 and CD40LG may contribute to the survival benefit of patients in the immune type A group.

\section{Introduction}

Since the development of immune checkpoint blockade cancer therapy, many clinical trials of immune checkpoint therapy combined with conventional targeted therapy against 
solid cancers have been performed, and this treatment has achieved great success in the cancer treatment field as a novel immunotherapy (1-3). With advances in clinical cancer immunotherapeutic regimens, closely associated tumor-related parameters have been intensively investigated. These parameters are thought to be linked to the efficacy of immune checkpoint blockade therapy and the prognosis of cancer patients (4-7). However, in the tumor microenvironment, there are many factors, such as genetic, immunological (cellular or humoral), and metabolic factors, that have been demonstrated to be involved in the immunosuppressive mechanism. For example, as cellular factors, regulatory effector $\mathrm{T}$ cells, myeloid-derived suppressor cells (MDSCs), tumor-associated macrophages (TAMs) and cancer-associated fibroblasts (CAFs) have been reported to exhibit protumor immunosuppressive actions (8-10).

Moreover, immune-type classifications that can contribute to the prediction of immune checkpoint blockade efficacy and the prognosis of cancer patients have been performed by several researchers using three main types of immunological features: PD-L1 expression level, tumor-infiltrating lymphocyte (TIL) status and tumor mutational burden (TMB) (11-16). PD-L1 is a major immune checkpoint molecule that is expressed on tumor cells or associated macrophages and is supposed to inhibit activated $\mathrm{T}$ cell function via PD-1/PD-L1 binding $(17,18)$. Meanwhile, some researchers have demonstrated that the simple combination of $\mathrm{PD}-\mathrm{L}^{+}$and $\mathrm{TIL}^{+}\left(\mathrm{CD}^{+}\right)$may predict a good response to immune checkpoint blockade $(11,12)$. Others have reported that TMB is a genuine biomarker for the prediction of immune checkpoint blockade efficacy (14).

Previously, our group performed an immunological classification based on PD-L1 and CD8B gene expression levels and demonstrated that the PD-L1+ and CD8B+ groups were associated with the upregulation of cytotoxic $\mathrm{T}$ lymphocyte (CTL) killing-associated genes, $\mathrm{T}$ cell activation genes, antigen-presentation genes and dendritic cell (DC) maturation genes, and promoted Thelper 1 (Th1) antitumor responses (19). However, there are few immune-type classification studies that directly evaluated cancer patient prognosis.

In the present study, we verified that the PD- $\mathrm{L}^{+} \mathrm{CD}^{+} \mathrm{B}^{+}$ group (type A) was associated with a better prognosis [5-year overall survival time (OST)] than the other types. In addition, we identified prognostic factors responsible for the survival benefit of patients in type A based on 293 immune response-associated gene expression datasets.

\section{Materials and methods}

Patient characteristics and study design. The Shizuoka Cancer Center launched Project HOPE in 2014 using multiomics analyses including whole exome sequencing (WES) and gene expression profiling (GEP). Ethical approval for the HOPE study was obtained from the Institutional Review Board of Shizuoka Cancer Center (authorization no. 25-33). In total, 1,763 patients with tumors were enrolled until March 2016 and the survival time was observed up to July 2019.

Clinical specimens. Tumor tissue samples weighing more than $0.1 \mathrm{~g}$ and with a tumor content greater than $50 \%$ were dissected along with surrounding normal tissue samples by pathologists.
GEP and WES analysis. DNA and RNA isolation and the GEP and WES analyses were performed as described previously (20). RNA samples with an RNA integrity number $\geq 6.0$ were used for microarray analysis. Labeled samples were hybridized to the SurePrint G3 Human Gene Expression 8x60 K v2 Microarray (Agilent Technologies). Microarray analysis was performed in accordance with the MIAME guidelines. For DNA data analysis, somatic mutations were identified by comparing data from tumor and corresponding blood samples. Mutations in 138 known driver genes were defined as those identified as pathogenic in the ClinVar database. Vogelstein et al (21) demonstrated that 138 genes, when altered by intragenic mutations, can promote or drive tumorigenesis. A most of tumors including colorectal cancers contain two to eight of these 'driver gene' mutations and the remaining mutations are passengers that do not contribute to tumorigenesis directly. Thus, these 138 driver mutations are accepted as relevant genes to the tumorigenesis (21). Single nucleotide variants (SNVs) of the total exonic mutations for each sequenced tumor included nonsynonymous, synonymous, and indel/frameshift mutations.

Renewal of the immune response-associated gene panel. The immune response-associated gene panel was described previously (22). In the present study, the gene panel was renewed by adding 119 immunological genes (293-gene panel) as shown in Table I. The panel consisted of 114 antigen-presenting cell (APC), T cell and natural killer cell receptor (NKR) genes; 48 cytokine signal and metabolic genes; 48 tumor necrosis factor (TNF) and TNF receptor superfamily genes; 23 regulatory T cell-associated genes; and 60 IFN-g pathway genes.

Statistical analysis. Based on the expression levels of the PD-L1 and CD8B genes, we classified all 1,763 tumors enrolled in the HOPE project into 4 immune types: type $\mathrm{A}, \mathrm{PD}-\mathrm{L1}^{+} \mathrm{CD}^{+} \mathrm{B}^{+}$; type $\mathrm{B}, \mathrm{PD}-\mathrm{L1}^{+} \mathrm{CD} 8$; type $\mathrm{C}, \mathrm{PD}-\mathrm{L1}^{-} \mathrm{CD} 8 \mathrm{~B}$; and type $\mathrm{D}$, $\mathrm{PD}-\mathrm{L}^{-} \mathrm{CD}^{-} \mathrm{B}^{+}$as described previously. A comparative analysis of the survival times between group $\mathrm{A}$ and the other groups was performed using the Kaplan-Meier method and Cox proportional hazards regression model. The upregulated genes derived from the 293-immune response-associated gene panel between tumor microenvironment (TME) immune type A and other types were identified using the volcano plot method with Benjamini-Hochberg correction. Upregulated immune response-associated genes with $>2$-fold expression differences $(\mathrm{P}<0.05)$ were identified. The heatmap expression data of upregulated genes in the immune type A group were investigated using GeneSpring GX software version 13.1.1 (Agilent Technologies). The association of upregulated gene expression levels with the OST was examined using the Kaplan-Meier method. A comparative analysis of the survival times between patients with low expression (less than the median) and patients with high expression (more than the median) of the identified genes in group type A (referred as to group A) was performed by the log-rank test using EZR software and Microsoft Excel. Regarding probable prognosis-associated genes identified in group A, the significance of these genes was analyzed using a multivariate Cox proportional hazards regression model with EZR software (23). Values of $\mathrm{P}<0.05$ denoted statistically significant differences. 


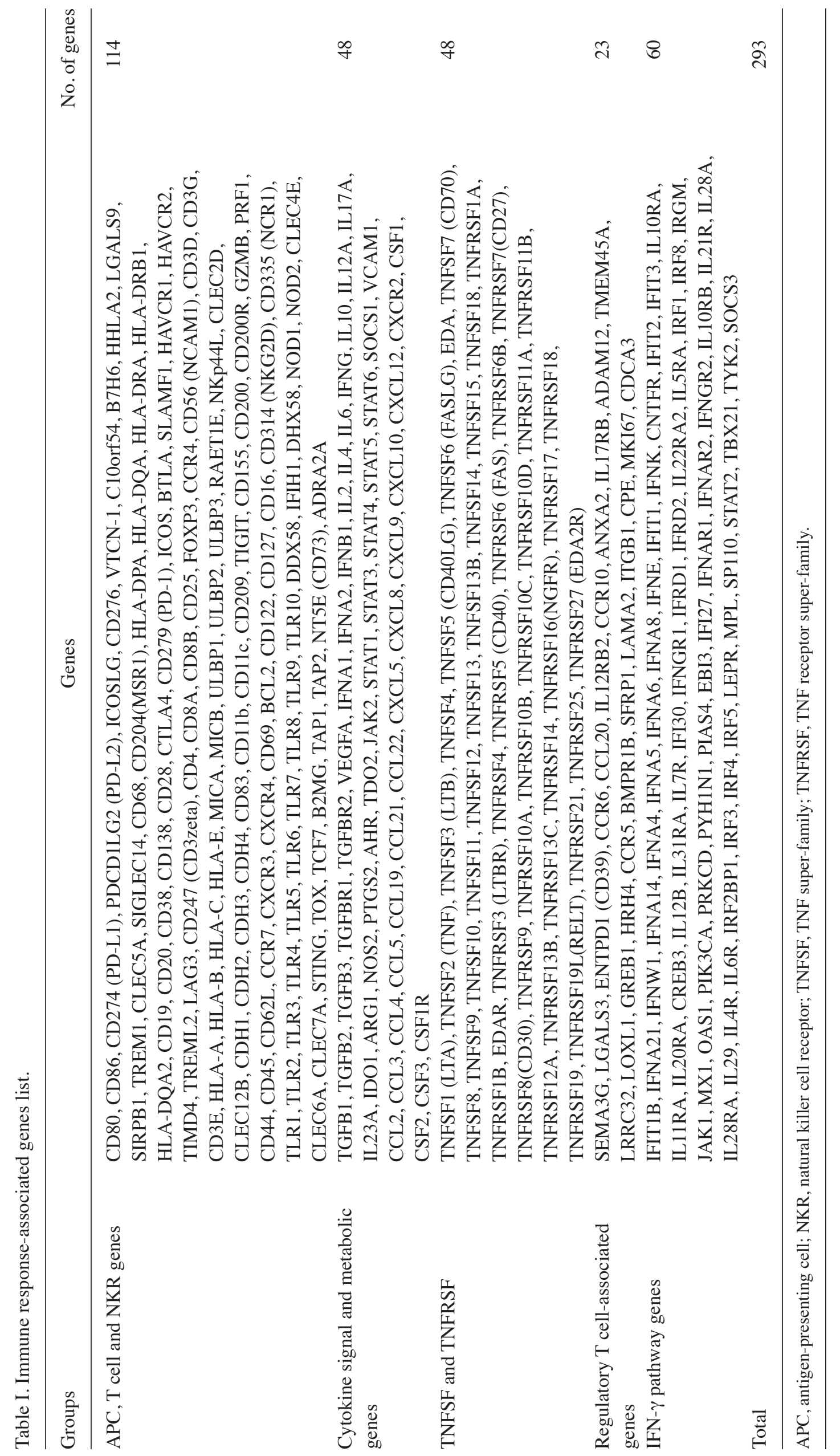


Table II. Association of immunological and genetic features with overall survival.

\begin{tabular}{llr}
\hline Group & \multicolumn{1}{c}{ Cohort (case no./5yrOS) } & \\
\hline Genetic mutations & & \\
Vogelstein & MT $(1084 / 77.3 \%)$ vs. WT $(679 / 74.6 \%)$ & 0.184 \\
TP53 & MT $(729 / 74.5 \%)$ vs. WT $(1034 / 77.4 \%)$ & 0.206 \\
KRAS & MT $(299 / 77.7 \%)$ vs. WT $(1464 / 75.9 \%)$ & 0.431 \\
EGFR & MT $(107 / 73.7 \%)$ vs. WT $(1656 / 76.3 \%)$ & 0.215 \\
PIK3CA & MT $(169 / 80.5 \%)$ vs. WT $(1594 / 75.9 \%)$ & 0.625 \\
BRAF & MT $(64 / 77.1 \%)$ vs. WT $(1699 / 76.2 \%)$ & 0.912 \\
TMB number & $>20(83 / 81.7 \%)$ vs. <20 $(1679 / 76.0 \%)$ & 0.512 \\
Gene amplification ${ }^{\mathrm{a}}$ & & \\
All 64 genes ${ }^{\mathrm{b}}$ & Yes $(575 / 75.9 \%)$ vs. No $(833 / 75.9 \%)$ & 0.858 \\
EGFR & Yes $(61 / 75.4 \%)$ vs. No $(1347 / 75.9 \%)$ & 0.746 \\
HER2 & Yes $(33 / 71.3 \%)$ vs. No $(1375 / 76.0 \%)$ & 0.530 \\
\hline
\end{tabular}

${ }^{\mathrm{a}}$ Gene amplification, fold-change in expression $\geq 5$ and copy number $\geq 6$. ${ }^{\mathrm{b}} 64$ amplified gene list was reported previously (20). Comparison of MST between cohorts was performed using the log-rank test. $\mathrm{P}<0.05$ denote statistically significant differences. 5 yrOS, 5 -year overall survival rate; MT, mutated; WT, wild-type; TMB, tumor mutation burden number.

\section{Results}

Association of the overall survival time with immune types. The 1,763 pairs of tumors and adjacent normal tissues derived from different cancer types were classified into 4 immune types based on the expression levels of the PD-L1 and CD8B genes. The patient numbers with different cancer types were described previously (17). The proportions of TME immune types A, B, C and D were 39.3, 26.5, 19.1 and 15.1\%, respectively. Survival time analysis at 5 years revealed that group A had a better prognosis than the other groups [5 year survival rate (\%); A (80.5) vs. B (73.9), C (73.4) and D (72.6), P=0.0005] (Fig. 1).

Association of genetic mutations and immunological surface markers with overall survival. The characteristics of genetic mutations, including Vogelstein driver mutations and SNVs, and gene amplification were described previously (19). The association of the genetic mutation status of driver gene mutations, such as TP53, KRAS, EGFR, PIK3CA and BRAF mutations, or gene amplification with the OST was investigated using the log-rank test. There was no significant association of genetic parameters with the OST (Table II).

The identification of upregulated immune response-associated genes in immune type Acompared with the other types. Based on the expression profile of the 293-immune response-associated gene panel, 62 upregulated immune response-associated genes (more than 2-fold and P-value 1.0E-50) were identified using volcano plots (Fig. 2).

Comparison of upregulated genes among immune types or between the poor prognosis and good prognosis cohorts. The heatmap expression data of 62 upregulated genes in group A were compared with those of the other groups. Interestingly, in group A, T cell effector activation genes (CXCL9, CXCL10, and TNFRSF9) and CTL killing genes (GZMB, CD16) showed high expression, while immune checkpoint genes such as CTLA4 and TIGIT also showed high expression levels. In contrast, in group C, T cell effector activation genes (ICOS, CD69, and CD40LG) and Th1 cytokine genes (IFNG and TNF) exhibited low expression (Fig. 3). Additionally, the upregulated $\mathrm{T}$ cell activation genes identified in group $\mathrm{A}$ showed a tendency of higher expression levels in the better survival cohort than in the poorer survival cohort, as shown in Fig. 4.

Association of the upregulated gene expression level with the overall survival time. The association of 62 upregulated genes in group A with the OST was analyzed by the log-rank test using EZR software. Ultimately, 18 genes were found to be significantly associated with prognosis (Table III). Memory $\mathrm{T}$ cell markers such as PD-1, CD27 and ICOS, as well as activated effector T cell genes (GZMB, CXCL10 and CD40LG) and mature DC marker genes (CD80 and SLAMF1), were selected as prognostic factors. Interestingly, immune checkpoint marker genes, such as HAVCR2 and TIGIT, were also verified as prognostic markers; however, the HAVCR2 gene was demonstrated to be a poor prognostic marker, although it was upregulated in group A.

Identification of probable prognostic genes using multivariate Cox hazards regression analysis. To evaluate the prognostic value of the genes, 18 probable prognostic genes identified using the Kaplan-Meier method from 62 upregulated genes in group A were analyzed by the Cox proportional hazards regression model. In particular, the multivariate analysis demonstrated that four upregulated genes, namely, GZMB, HAVCR2, CXCL9 and CD40LG, maintained their significance $(\mathrm{P}<0.05)$, as shown in Table IV. The survival curves of these four significant genes were drawn with the Kaplan-Meier method, and the OST was compared between the group that was higher-than-the median-level and the group 

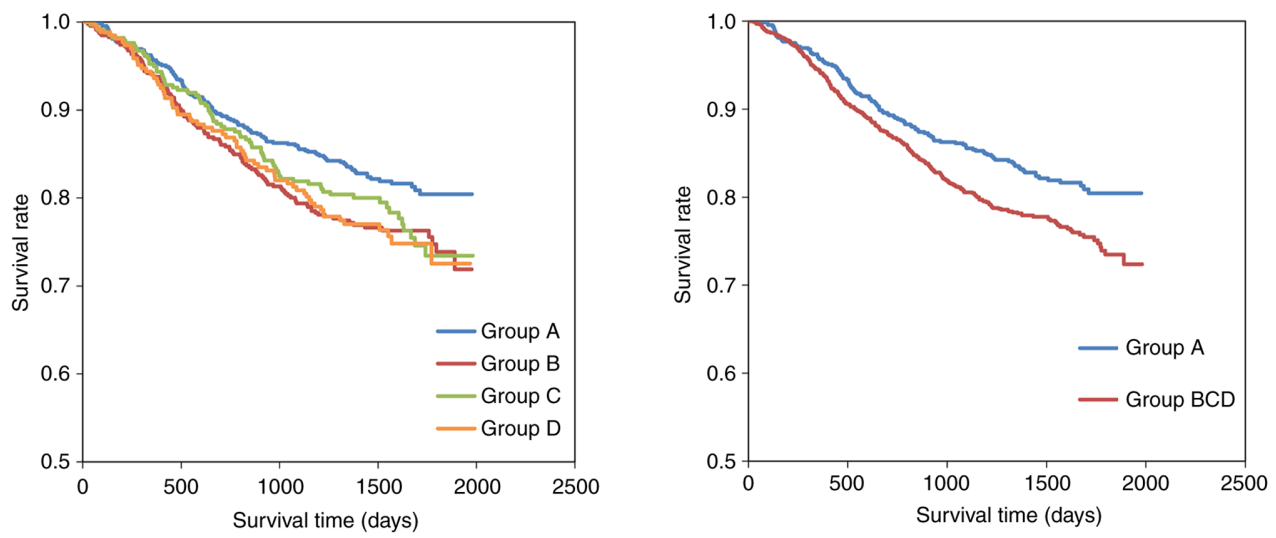

\begin{tabular}{lllll}
\hline & $\mathrm{N}$ & $5 \mathrm{yrOS}(\%)$ & $\mathrm{HR}(95 \% \mathrm{Cl})$ & $P$ value \\
\hline $\mathrm{A}$ & 692 & $80.5 \%$ & Ref & Ref \\
B & 467 & $73.9 \%$ & $1.386(1.075-1.788)$ & $0.0119^{*}$ \\
$\mathrm{C}$ & 337 & $73.4 \%$ & $1.278(0.960-1.699)$ & 0.0922 \\
$\mathrm{D}$ & 267 & $72.6 \%$ & $1.414(1.048-1.909)$ & $0.0234^{*}$ \\
\hline$--9 C D$ & 1071 & $73.5 \%$ & $1.358(1.097-1.683)$ & $0.005^{* *}$ \\
\hline
\end{tabular}

Figure 1. Evaluation of the OS time in 1,763 patients with cancer registered in the HOPE project. Survival time analysis at 5 years revealed that group A had a better prognosis than the other groups. A comparative analysis of the survival times between group A and the other groups was performed using the Kaplan-Meier method and Cox proportional hazards regression model. The OS analysis indicated a significant survival benefit at 5 years for group A. ${ }^{*} \mathrm{P}<0.05$ and ${ }^{* * *} \mathrm{P}<0.01$. Number of cases in group $A(n=692)$, group $B(n=467)$, group $C(n=337)$ and group $D(n=267)$. The number of cases with different types of cancer was as follows: 107 breast, 601 colorectal, 27 skin, 25 esophageal, 248 stomach, 49 uterine and ovarian, 69 bile duct and pancreatic, 152 head and neck, 98 liver, 4 brain, 14 bone, 348 lung and 21 kidney cancer. OS, overall survival; HR, hazard ratio; HOPE, High-tech Omics-based Patient Evaluation.

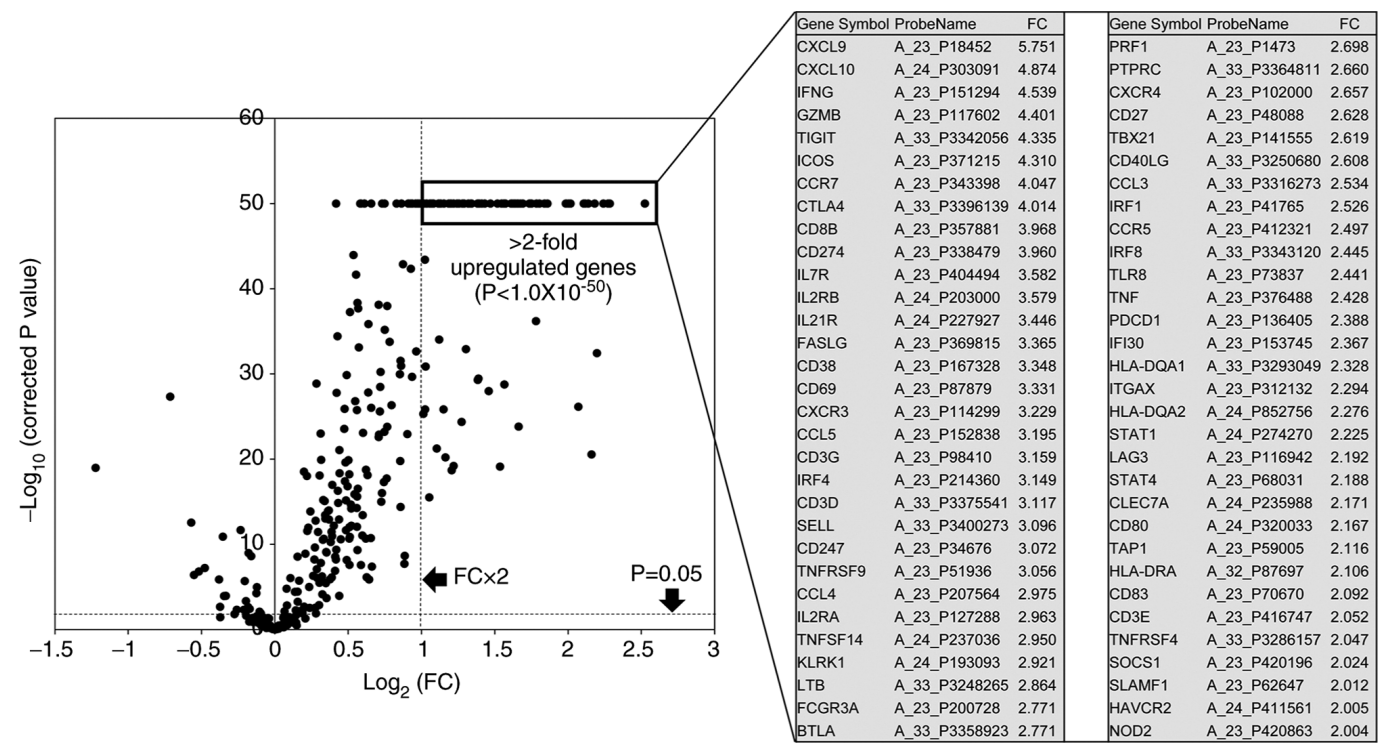

Figure 2. Identification of upregulated immune response-associated genes in tumor microenvironment immune type A compared with other types. A total of 62 upregulated immune response-associated genes with $>2$-fold expression differences were identified using volcano plots with Benjamini-Hochberg correction. The upregulation of all listed genes denoted statistically significant differences $(\mathrm{P}<0.05)$. FC, fold-change.

that was lower-than-the median-level, as shown in Fig. 5. The upregulation of GZMB, CXCL9 and CD40LG gene expression might be linked to better prognosis in group A patients.

\section{Discussion}

With advances in genome analysis technologies such as NGSand single-cell RNA sequencing, probable immunological factors belonging to the TME and associated with prognosis have been more intensively, specifically and accurately investigated (24-26). Beyond the already-known TME factors that might be responsible for the efficacy of cancer immunotherapy, such as positive PD-L1 expression, a high mutational burden and an advanced TIL status, more specific and dynamic biomarkers associated with the immune response have been reported (27-29). Recently, Kumagai et al demonstrated using cytometry by time of flight (CyTOF) analysis based on single-cell RNA-seq that a balance between 


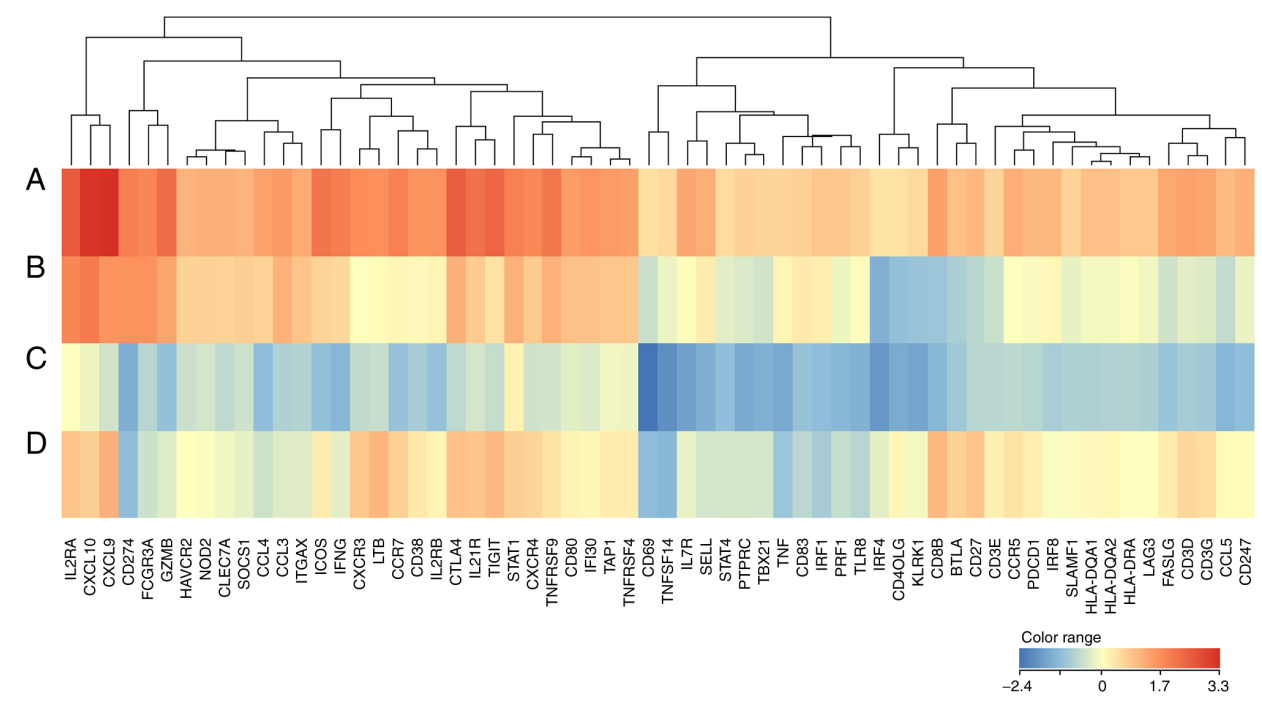

Figure 3. Hierarchical clustering analysis of 62 upregulated genes in each immune group. Each row in the matrix represents the expression level of a gene feature in an individual group. The red and blue colors in the panel reflect the relative expression level of the gene, as indicated in the color scale ( $\log _{2}$-transformed scale). Group A ( $\mathrm{n}=692)$, group B $(\mathrm{n}=467)$, group C $(\mathrm{n}=337)$ and group $\mathrm{D}(\mathrm{n}=267)$.

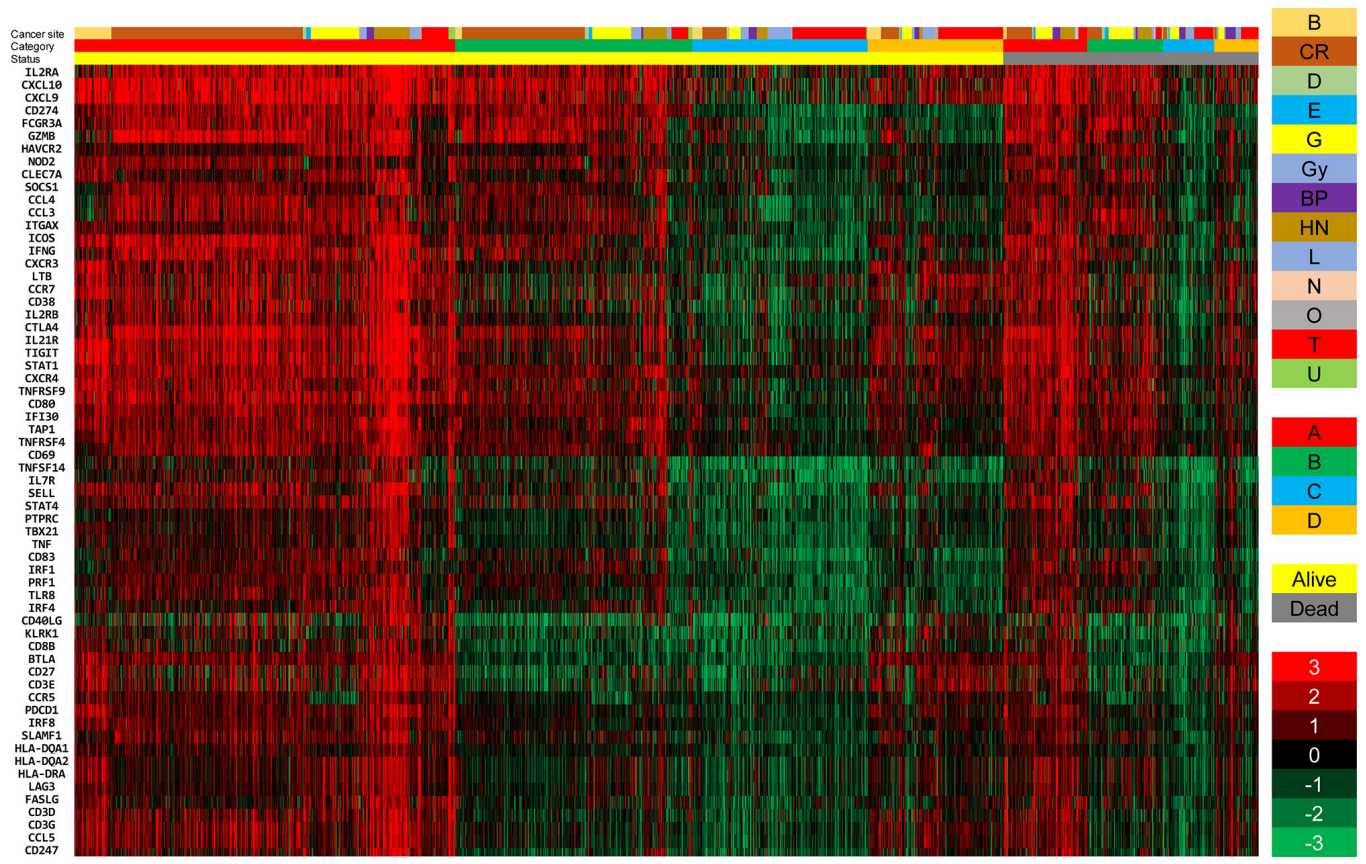

Figure 4. Comparison of the expression levels of 62 upregulated genes between alive and deceased patients with cancer. The two cohorts of patients were divided into 4 immune groups, and classified into 13 histological types. The data are presented in matrix format, where each row represents an individual case, and each column represents a gene. Each cell in the matrix represents the expression level of a gene in an individual case. The red and green colors reflect the gene expression levels, as indicated in the color scale ( $\log _{2}$-transformed scale) in the bottom right corner. B, breast; CR, colorectal; D, skin; E, esophageal; G, stomach; Gy, uterine and ovarian; BP, bile duct and pancreatic; HN, head and neck; L, liver; N, brain; O, bone; T, lung; and U, kidney.

$\mathrm{PD}-1^{+} \mathrm{CD} 8^{+} \mathrm{T}$ cells and $\mathrm{PD}-1^{+} \mathrm{CD} 4^{+} \mathrm{FoxP} 3^{+}$Treg cells is a critical determinant of the response to anti-PD-1/PD-L1 blockade therapy (29).

Previously, we reported an efficient immunological classification based on PD-L1 and CD8B gene expression levels and demonstrated that immune type $\mathrm{A}\left(\mathrm{PD}-\mathrm{Ll}^{+} \mathrm{CD} 8 \mathrm{~B}^{+}\right)$was associated with the Th1 T cell and NK cell activation pathways, dendritic cell maturation and cancer-apoptosis activation signals and showed the highest score in immune-activation signaling pathways by means of Ingenuity Pathways Analysis
(IPA) software (19). Similar studies have been conducted that showed antitumor immunological features in PD-L1+CD8+ cohort $(11,12)$.

However, there have been few studies that have performed a long-term follow-up of overall survival in cancer patients belonging to the immune type classifications described above. Ock et al classified similarly solid tumors into specific immune types based on PD-L1 and CD8 gene expression data derived from The Cancer Genome Atlas (TCGA) database and compared the survival time between 
Table III. Probable prognostic genes identified from 62 upregulated genes.

\begin{tabular}{|c|c|c|c|c|}
\hline Probe name & Fold-change & Gene symbol & 5yrOS $(\%)^{\text {a }}$ Positive. vs. Negative & Log-rank P-value \\
\hline A_23_P117602 & 4.401 & GZMB & 80.7 vs. 71.7 & $1.44 \times 10^{-4}$ \\
\hline A_24_P411561 & 2.005 & HAVCR2 & 74.1 vs. 78.3 & $2.03 \times 10^{-3}$ \\
\hline A_23_P371215 & 4.31 & ICOS & 80.5 vs. 71.9 & $2.14 \times 10^{-3}$ \\
\hline A_23_P18452 & 5.751 & CXCL9 & 80.5 vs. 71.8 & $3.06 \times 10^{-3}$ \\
\hline A_23_P420196 & 2.024 & SOCS1 & 79.6 vs. 72.8 & $3.44 \times 10^{-3}$ \\
\hline A_23_P136405 & 2.388 & PDCD1 & 80.3 vs. 72.1 & $3.6 \times 10^{-3}$ \\
\hline A_24_P303091 & 4.874 & CXCL10 & 80.5 vs. 71.9 & $4.76 \times 10^{-3}$ \\
\hline A_23_P98410 & 3.159 & CD3G & 79.8 vs. 72.7 & $1.47 \times 10^{-2}$ \\
\hline A_23_P420863 & 2.004 & NOD2 & 79.1 vs. 73.3 & $1.82 \times 10^{-2}$ \\
\hline A_33_P3250680 & 2.608 & CD40LG & 78.6 vs. 74.0 & $2.52 \times 10^{-2}$ \\
\hline A_33_P3375541 & 3.117 & CD3D & 79.7 vs. 72.7 & $2.6 \times 10^{-2}$ \\
\hline A_23_P62647 & 2.012 & SLAMF1 & 79.7 vs. 72.6 & $2.6 \times 10^{-2}$ \\
\hline A_24_P320033 & 2.167 & CD80 & 79.2 vs. 73.2 & $2.96 \times 10^{-2}$ \\
\hline A_23_P48088 & 2.628 & $\mathrm{CD} 27$ & 79.9 vs. 72.7 & $3.31 \times 10^{-2}$ \\
\hline A_23_P416747 & 2.052 & CD3E & 78.9 vs. 73.6 & $3.64 \times 10^{-2}$ \\
\hline A_33_P3342056 & 4.335 & TIGIT & 79.3 vs. 73.2 & $4.16 \times 10^{-2}$ \\
\hline A_23_P338479 & 3.96 & CD274 & 78.6 vs. 73.8 & $4.33 \times 10^{-2}$ \\
\hline A_23_P41765 & 2.526 & IRF1 & 79.2 vs. 73.0 & $4.53 \times 10^{-2}$ \\
\hline
\end{tabular}

${ }^{a}$ The 5yrOS between positive (higher expression than the median level) and negative (lower expression than the median level) groups were compared using the log-rank test using EZR software. Ultimately, 18 genes were found to be significantly associated with prognosis of patients with cancer. Only the HAVCR2 gene demonstrated a negative association with prognosis. 5yrOS, 5-year overall survival.

Table IV. Cox proportional hazards regression analysis of overall survival in upregulated genes.

\begin{tabular}{lll}
\hline Variable & Hazard ratio $(95 \% \mathrm{CI})$ & P-value \\
\hline GZMB & $0.628(0.496-0.795)$ & $1.11 \times 10^{-4}$ \\
HAVCR2 & $1.848(1.479-2.309)$ & $6.63 \times 10^{-8}$ \\
CXCL9 & $0.778(0.613-0.988)$ & 0.0393 \\
CD40LG & $0.792(0.642-0.977)$ & 0.0292 \\
\hline
\end{tabular}

From probable prognosis-associated genes identified in group A, the significance of those genes was analyzed using multivariate Cox proportional hazards regression model in the EZR software. $\mathrm{P}<0.05$ denoted statistically significant differences.

immune types; however, the temporary difference in 3-year survival time in type A finally disappeared in the 5-year comparison (12).

In the current study, we followed 1,763 patients with tumors up to 70 months after registration in the project HOPE study. Survival time analysis at 5 years revealed that group A had a better prognosis than the other groups, as shown in Fig. 1. There are some concerns regarding the temporary results of the present survival analysis: i) Miscellaneous cancer patients across various histology groups were included, and ii) there were various clinical courses, including different types of therapies and response statuses. However, despite different clinical courses in individual patients, the immunological status at cancer diagnosis can be determined temporarily in terms of the OST, and could be a reference parameter for therapeutic design because some immunological mechanisms are involved in tumor regression after or even during chemo- and radiation therapy (30-33).

In the present study, the impressive findings were that memory $\mathrm{T}$ cell markers (central $\sim$ effector memory), such as PD-1, CD27 and ICOS, were selected as prognostic factors. In addition to effector-activated CTLs and NK cells, memory marker ${ }^{+} \mathrm{T}$ cells should be considered crucial factors because i) $\mathrm{PD}-1^{+} \mathrm{T}$ cells can achieve a good balance between good and poor responses by immune checkpoint blockade (27), and ii) effector memory $\mathrm{T}$ cells that proliferate by the stimulation of antigen-presenting cells, can be differentiated into activated effector CTLs (34). Another important observation was that $\mathrm{T}$ cell exhaustion marker genes such as HAVCR22 (TIM3) and TIGIT were included as prognostic markers. However, HAVCR2 was found to be a negative prognostic marker, suggesting that it did not contribute to the good prognosis of patients in immune group A. Very recently, Simon et al demonstrated that a high frequency of the PD- ${ }^{+} \mathrm{TIGIT}^{+}$ (double-positive) $\mathrm{CD}^{+} \mathrm{T}$ cell subset in peripheral blood can be a good predictive marker for a good response to anti-PD-1 therapy (35). Therefore, these cells should be prolonged by anti-PD-1/PD-L1 blockade to maintain the antitumor effect, which could contribute to the good prognosis in cancer patients belonging to immune type A.

Additionally, based on prognostic factor profiling in immune group A, the upregulation of the CD80, CD274 and SLAMF1 (36) genes might suggest the presence of mature dendritic cells in the TME. Interestingly, Schetters et al demonstrated that anti-PD-1 immune checkpoint blockade induced mature monocyte-derived 

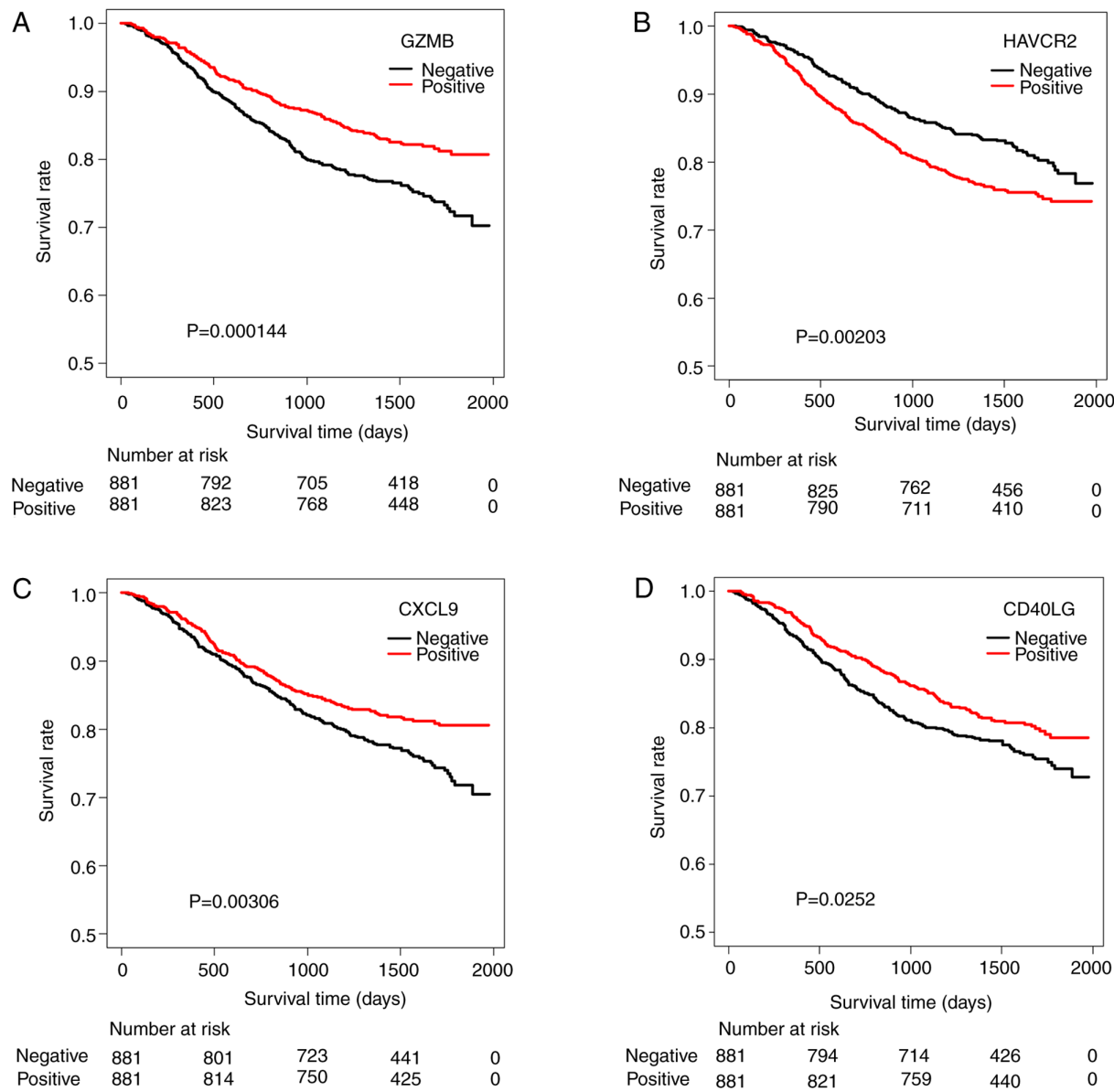

Figure 5. Survival curves of 4 significant prognostic genes identified using the Cox hazards regression model. Overall survival time between patients with positive (higher expression than the median level of an individual gene) and negative expression (lower expression than the median level of an individual gene) was compared by the log-rank test using EZR software. (A) GZMB, (B) HAVCR2, (C) CXCL9 and (D) CD40LG.

dendritic cells in the TME (37), which means that the presence of mature dendritic cells in the tumor site could be a key factor in the prediction of ICB efficacy.

Considering that immunological conditions are varied and complicated in the TME, the status of patients with cancer is volatile and undetermined before the start of treatment. Most likely, immune type group A (PD- $\left.\mathrm{L}^{+} \mathrm{CD}^{+}\right)$could be a good candidate to elicit neoantigen-specific $\mathrm{T}$ cell reactions and result in an improved prognosis in cancer patients. Efficient combination therapy with chemo- and radiation therapy should be explored for these types of cancer cohorts in the future.

\section{Acknowledgements}

Not applicable.

\section{Funding}

No funding was received.

\section{Availability of data and materials}

The datasets generated and/or analyzed during the current study are available in the National Bioscience Database Center repository (accession no. hum0127; https://humandbs. biosciencedbc.jp/en/).

\section{Authors' contributions}

RK and YA participated equally in the design of the study and drafting of the manuscript, and were responsible for completing the study. AS, YO, MTe, KUe, TO, SN, YH, NH, YK, YT, HKat, MNi, KT, HKas, MNa and YI were responsible for the clinical work, including the collection of clinical samples. TN, YS, KUr, KO, AI, HM, CM, AK, KW and TA participated in the design of the experiments and performed the genetic analysis. TS contributed to the pathological diagnosis. AN and KM contributed to data analysis and interpretation and confirmed the authenticity of all the raw data. MTa, HKe and KY designed the current study, revised the manuscript critically for important intellectual content and gave final approval of the version to be published by taking responsibility for the content. All authors read and approved the final manuscript.

\section{Ethics approval and consent to participate}

The Shizuoka Cancer Center launched Project HOPE based on multiomics analyses, including WES and GEP. Ethics approval for the HOPE study was obtained from the institutional review board at the Shizuoka Cancer Center (authorization no. 25-33). Written informed consent was obtained from all patients enrolled in the study. 


\section{Patient consent for publication}

Written informed consent was obtained from all patients for the publication of any associated data and accompanying images.

\section{Competing interests}

The authors declare that they have no competing interests.

\section{References}

1. Weber JS, O'Day S, Urba W, Powderly J, Nichol G, Yellin M, Snively J and Hersh E: Phase I/II study of ipilimumab for patients with metastatic melanoma. J Clin Oncol 26: 5950-5956, 2008.

2. Topalian SL, Hodi FS, Brahmer JR, Gettinger SN, Smith DC, McDermott DF, Powderly JD, Carvajal RD, Sosman JA, Atkins MB, et al: Safety, activity, and immune correlates of anti-PD-1 antibody in cancer. N Engl J Med 366: 2443-2454, 2012

3. Brahmer JR, Tykodi SS, Chow LQ, Hwu WJ, Topalian SL, Hwu P, Drake CG, Camacho LH, Kauh J, Odunski K, et al: Safety and activity of anti-PD-L1 antibody in patients with advanced cancer. N Engl J Med 366: 2455-2465, 2012.

4. Ascierto RA, Capone M, Urba WJ, Bifuco CB, Botti G, Lugli A Marincola FM, Ciliberto G, Galon J and Fox BA: The additional facet of immunoscore: Immunoprofiling as a possible predictive tool for cancer treatment. J Transl Med 11: 54, 2013.

5. Gnjatic S, Bronte V, Brunet LR, Butler MO, Disis ML, Galon J, Hakansson LG, Hanks BA, Karanikas V, Khleif SN, et al: Identifying baseline immune-related biomarkers to predict clinical outcome of immunotherapy. J Immunother Cancer 5: 44, 2017.

6. Johnson DB, Frampton GM, Rioth MJ, Yusko E, Xu Y, Guo X, Ennis RC, Fabrizio D, Chalmers ZR, Greenbowe J, et al: Targeted next generation sequencing identifies markers of response to PD-1 blockade. Cancer Immunol Res 4: 959-967, 2016.

7. Dudley JC, Lin MT, Le DT and Eshleman JR: Microsatellite instability as a biomarker for PD-1 blockade. Clin Cancer Res 22 813-820, 2016.

8. Yarchoan M, Johnson BA III, Lutz ER, Laheru DA and Jaffee EM: Targeting neoantigen to augment antitumor immunity. Nat Rev Cancer 17: 209-222, 2017.

9. Lin $\mathrm{Y}, \mathrm{Xu} \mathrm{J}$ and Lan H: Tumor-associated macrophages in tumor metastasis: Biological roles and clinical therapeutic applications. J Hematol Oncol 12: 76, 2019.

10. Fleming B, Hu X, Weber R, Nagibin V, Groth C, Artevogt P, Utical J and Umansky V: Targeting myeloid-derived suppressor cells to bypass tumor-induced immunosuppression. Front Immunol 9: 398, 2018.

11. Rooney MS, Shukla SA, Wu CJ, Getz G and Hacohen N: Molecular and genetic properties of tumors associated with local immune cytolytic activity. Cell 160: 48-61, 2015.

12. Ock CY, Keam B, Kim S, Lee JS, Kim M, Kim TM, Jeon YK, Kim DW, Chung DH and Heo DS: Pan-cancer immunogenic perspective on the tumor microenvironment based on PD-L1 and CD8 T-cell infiltration. Clin Cancer Res 22: 2261-2270, 2016.

13. Teng MW, Ngiow SF, Ribas A and Smyth MJ: Classifying cancers based on T-cell infiltration and PD-L1. Cancer Res 75: $2139-2145,2015$

14. Yarchoan M, Hopkins A and Jaffee EM: Tumor mutational burden and response rate to PD-1 inhibition. N Engl J Med 377: 2500-2501, 2017

15. Bruni D, Angell HK and Galon J: The immune contexture and immunoscore in cancer prognosis and therapeutic efficacy. Nat Rev Cancer 20: 662-680, 2020.

16. Snyder A, Makarov V, Merghoub T, Yuan J, Zaretsky JM Desrichard A, Walsh LA, Postow MA, Wong P, Ho TS, et al: Genetic basis for clinical response to CTLA-4 blockade in melanoma. N Engl J Med 371: 2189-2199, 2014.

17. Iwai Y, Ishida M, Tanaka Y, Okazaki T, Honjo T and Minato N Involvement of PD-L1 on tumor cells in the escape from host immune system and tumor immunotherapy by PD-L1 blockade. Proc Natl Acad Sci USA 99: 12293-12297, 2002.

18. Petty AJ, Dai R, Lapalombella R, Baiocchi RA, Benson DM, Li Z, Huang X and Yang Y: Hedgehog-induced PD-L1 on tumor-associated macrophages is critical for suppression of tumor-infiltrating $\mathrm{CD}^{+} \mathrm{T}$ cell function. JCI Insight 6: e146707, 2021.
19. Kondou R, Iizuka A, Nonomura C, Miyata H, Ashizawa T, Nagashima T, Ohshima K, Urakami K, Kusuhara M, Yamaguchi K and Akiyama Y: Classification of tumor microenvironment immune types based on immune response-associated gene expression. Int J Oncol 54: 219-228, 2019.

20. Ohshima K, Hatakeyama K, Nagashima T, Watanabe Y, Kanto K, Doi Y, Ide T, Shimoda Y, Tanabe T, Ohnami S, et al: Integrated analysis of gene expression and copy number identified potential cancer driver genes with amplification-dependent overexpression in 1,454 solid tumors. Sci Rep 7: 641, 2017.

21. Vogelstein B, Papadopoulos N, Velculescu VE, Zhou S, Doaz LA Jr and Kinzler KW: Cancer genome landscapes. Science 339: 1546-1558, 2013.

22. Akiyama Y, Kondou R, Iizuka A, Ohshima K, Urakami K, Nagashima T, Shimoda Y, Tanabe T, Ohnami S, Ohnami S, et al: Immune response-associated gene analysis of 1,000 cancer patients using whole-exome sequencing and gene expression profiling-Project HOPE. Biomed Res 37: 233-242, 2016.

23. Kanda Y: Investigation of the freely available easy-to-use software 'EGR' for medical statistics. Bone Marrow Transplant 48: 452-458, 2013.

24. Gubin MM, Esaulova E, Ward JP, Malkova ON, Runci D, Wong P, Noguchi T, Arthur CD, Meng W, Alspach E, et al: High-dimensional analysis delineates myeloid and lymphoid compartment remodeling during successful immune-checkpoint cancer therapy. Cell 175: 1014-1030, 2018.

25. Guo X, Zhang Y, Zheng L, Zheng C, Song J, Zhang Q, Kang B, Liu Z, Jin L, Xing R, et al: Global characterization of T cells in non-small-cell lung cancer by single-cell sequencing. Nat Med 24: 978-985, 2018.

26. Sathe A, Grimes SM, Lau BT, Chen J, Suarez C, Huang RJ, Poultsides G and Ji HP: Single-cell genomic characterization reveals the cellular reprogramming of the gastric tumor microenvironment. Clin Cancer Res 26: 2640-2653, 2020.

27. Hossain MA, Liu G, Dai B, Si Y, Yang Q, Wazir J, Birnbaumer L and Yang Y: Reinvigorating exhausted CD8+ cytotoxic T lymphocytes in the tumor microenvironment and current strategies in cancer immunotherapy. Med Res Rev 41: 156-201, 2021.

28. Xiao Z, Locasale JW and Dai Z: Metabolism in the tumor microenvironment: Insights from single-cell analysis. Oncoimmunology 9: 1726556,2020

29. Kumagai S, Togashi Y, Kamada T, Sugiyama E, Nishinakamura H, Takeuchi Y, Vitaly K, Itahashi K, Maeda Y, Matsui S, et al: The PD-1 expression balance between effector and regulatory $\mathrm{T}$ cells predicts the clinical efficacy of PD-1 blockade therapies. Nat Immunol 21: 1346-1358, 2020

30. Opzoomer JW, Sosnowska D, Anstee J, Spicer JF and Arnold JN: Cytotoxic chemotherapy as an immune stimulus: A molecular perspective on turning up the immunological heat on cancer. Front Immunol 10: 1654, 2019.

31. Yu WD, Sun G, Li J, Xu J and Wang X: Mechanisms and therapeutic potentials of cancer immunotherapy in combination with radiotherapy and/or chemotherapy. Cancer Lett 452: 66-70, 2019.

32. Turgeon GA, Weickhardt A, Azad AA, Solomon B and Siva S: Radiotherapy and immunotherapy: A synergistic effect in cancer care. Med J Aust 210: 47-53, 2019.

33. Rodriguez-Ruiz ME, Vanpouille-Box C, Melero I, Formenti SC and Demaria S: Immunological mechanisms responsible for radiation-induced abscopal effect. Trends Immunol 39: 644-655, 2018.

34. Mitsuya K, Akiyama Y, Iizuka A, Miyata H, Deguchi S, Hayashi N, Maeda C, Kondou R, Kanematsu A, Watanabe K, et al: Alpha-type-1 polarized dendritic cell-based vaccination in newly diagnosed high-grade glioma: A phase II clinical trial. Anticancer Res 40: 6473-6484, 2020.

35. Simon S, Voillet V, Vignard V, Wu Z, Dabrowski C, Jouand N, Beauvais T, Khammari A, Braudeau C, Josien R, et al: PD-1 and TIGIT coexpression identifies a circulating CD8 T cell subset predictive of response to anti-PD-1 therapy. J Immunother Cancer 8: e001631, 2020.

36. Bleharski JR, Niazi KR, Sieling PA, Cheng G and Modlin RL: Signaling lymphocytic activation molecule is expressed on $\mathrm{CD}_{40}$ ligand-activated dendritic cells and directly augments production of inflammatory cytokines. J Immunol 167: 3174-3181, 2001.

37. Schetters ST, Rodriguez E, Kruijssen LJ, Crommentuijn MHW, Boon L, Van den Bossche J, Den Haan JMM and Van Kooyk Y: Monocyte-derived APCs are central to the response of PD1 checkpoint blockade and provide a therapeutic target for combination therapy. J Immunother Cancer 8: e000588, 2020. 